\title{
Does Exhaustive Search for Microcalcifications Improve Diagnostic Yield in Stereotactic Core Needle Breast Biopsies?
}

\author{
Margaret M. Grimes, M.D., Lampros S. Karageorge, M.D., Jacquelyn P. Hogge, M.D. \\ Departments of Pathology (MMG, LSK) and Radiology (JPH), Virginia Commonwealth University, \\ Richmond, Virginia
}

\begin{abstract}
Stereotactic core needle biopsy (SCNB) of the breast is a cost-effective alternative to needle localization biopsy for the diagnosis of mammographic calcifications. We questioned whether an exhaustive search for calcium in the small samples obtained in SCNB yields more diagnostic information than that obtained with examination of a standard number of sections. We retrospectively reviewed 168 specimens from 123 patients with mammographic calcifications, including cases in which radiographic suspicion ranged from low to high. Microcalcifications were identified on three initial levels in 112 specimens. Additional sections were examined in $\mathbf{5 0}$ specimens. The final diagnosis differed from the diagnosis based on three levels in 11/50 cases (22\%). In 6/50 (12\%), complete sectioning yielded a specific diagnosis. The increase in technical cost associated with the additional levels was $414 \%$ per case. We conclude that exhaustive searching for microcalcifications in SCNB yields a small increase in specific diagnostic information and a high technical cost. In individual cases, the additional information may be critical for appropriate patient management.
\end{abstract}

KEY WORDS: Breast, Core needle biopsy, Microcalcifications.

Mod Pathol 2001;14(4):350-353

Stereotactic core needle biopsies (SCNB) are commonly employed in the diagnostic assessment of suspicious mammographic calcifications. The medical advantages and cost benefits of this procedure compared with needle localization biopsy (NLB)

Copyright () 2001 by The United States and Canadian Academy of Pathology, Inc.

VOL. 14, NO. 4, P. 350, 2001 Printed in the U.S.A.

Date of acceptance: September 8, 2000.

Presented in part at the United States-Canadian Academy of Pathology Annual Meeting, March 1998, Boston, Massachusetts.

Address reprint requests to: Margaret M. Grimes, M.D., Department of Pathology, Virginia Commonwealth University, Medical College of Virginia Campus, P.O. Box 980662, Richmond, VA 23298-0662; e-mail: mgrimes@hsc.vcu.edu; fax: 804-828-5055. have been demonstrated (1). Pathologic correlation with mammographic calcification in NLB is essential for accurate diagnosis. Postoperative radiographic confirmation of the presence of suspicious calcifications and their localization in the specimen is required to direct the pathologist in the proper sectioning of the tissue. Processing of all fibrous parenchymal tissue in NLB has been recommended for optimum diagnostic yield (2). Similarly, radiographic confirmation of the presence of calcifications in SCNB specimens is recommended to document that the suspicious area has been sampled (3). Because radiographically detected calcifications may be as small as $100 \mu \mathrm{m}$ (4), they are not always identified on initial sectioning of these biopsies, and additional sections may be required to locate them. Considering that similarly sized core needle biopsies of other organs are usually diagnosed on the basis of a standard number of histologic sections, we reviewed a series of SCNB to determine the incidence of changes in diagnosis associated with complete sectioning of SCNB to detect microcalcifications. We also determined the technical costs associated with the additional sections.

\section{MATERIALS AND METHODS}

We retrospectively reviewed all available SCNB cases performed over a 10-month period. The specimens were obtained using a 14-gauge needle and biopsy gun under stereotactic guidance. At our institution, three hematoxylin and eosin-stained levels of SCNB are routinely examined. These consist of three slides, with a ribbon of two to three $4-\mu \mathrm{m}$ thick sections of tissue on each slide. If examination of the three levels fails to reveal calcium phosphate $(\mathrm{CaP})$ in the tissue, the sections are examined by polarized light microscopy for the presence of calcium oxalate $(\mathrm{CaOx})$. If atypical ductal hyperplasia $(\mathrm{ADH})$ or carcinoma is identified on the first three levels, no further sectioning usually is performed 
even if no calcifications have been identified because either diagnosis leads to NLB or other appropriate surgical excision. In the absence of calcifications or a histologic diagnosis that would necessitate excision, the paraffin block is exhaustively sectioned, and every third level is stained and examined. The remaining levels are stained and examined if these additional levels are negative. We reviewed all stained sections in each case. In those cases in which additional sections beyond the routine levels had been prepared, we compared the histologic findings on the first three levels with the final diagnosis.

In those cases that were completely sectioned, both the mammograms (which in most cases included magnification views of the suspicious calcifications) and the specimen radiographs of the SCNB were also reviewed for this study. The location, number, and morphology of the calcifications in the mammograms were evaluated and described in accordance with the American College of Radiology Breast Imaging and Reporting Data System. The lesions were then graded $\mathrm{B}$ to $\mathrm{H}(\mathrm{B}=$ benign, $\mathrm{L}$ = low suspicion, $\mathrm{M}=$ medium suspicion, $\mathrm{H}=$ high suspicion). The specimen radiographs were reviewed for presence of calcifications in the specimen.

The technical cost associated with the preparation of the additional histologic sections versus standard levels was analyzed.

\section{RESULTS}

We reviewed 168 specimens from 123 patients with mammographic calcifications. In 23 specimens (14\%), three levels revealed atypical duct hyperplasia $(\mathrm{ADH})$ or carcinoma, either in situ or invasive (4 with $\mathrm{ADH}, 15$ with duct carcinoma in situ, 4 with invasive carcinoma). Microcalcifications were identified in 18/23 (78\%). In all 18 cases, these were CaP. The diagnosis made on the basis of three levels was benign with no atypia in 145 specimens. Microcalcifications were identified in 93/145 (64\%). These consisted of $\mathrm{CaP}$ in 89 (three of those cases also had $\mathrm{CaOx}$ ) and of $\mathrm{CaOx}$ in 4 cases.

Fifty specimens had been completely sectioned. This includes two cases in which the diagnosis on the first three levels was ADH and 12 in which $\mathrm{CaP}$ or $\mathrm{CaOx}$ was identified in our retrospective review of the first three levels. On average, 10 additional stained slides were prepared (range, 3-26). Calcifications were identified in the additional slides in 21 of the 50 specimens $(42 \%)$. The final diagnosis differed from that made on the basis of the first three slides in 11 cases (Table 1). In 6 of the 50 cases (12\%), complete sectioning yielded a specific diagnosis (Cases 1, 3, 6, 8, 9, and 11) including one case
TABLE 1. Changes in Diagnosis on Deeper Levels

\begin{tabular}{rll}
\hline Case & \multicolumn{1}{c}{ Initial Diagnosis } & \multicolumn{1}{c}{ Final Diagnosis } \\
\hline 1 & Fibroadipose tissue & Fibroadenoma \\
2 & Benign breast tissue, NPD & Fibrosis \\
3 & Fibrocystic changes & Fibroadenoma \\
4 & Fat, scant breast tissue & Fibrocystic changes \\
5 & ADH & DCIS \\
6 & Mild hyperplasia & ADH \\
7 & Fat, fibrous tissue & Benign breast tissue, NPD \\
8 & Fibrocystic changes & Papilloma (microscopic) \\
9 & Fibrosis & Fibroadenoma \\
10 & ADH & DCIS \\
11 & Fat, fibrous tissue & Fibroadenoma \\
\hline
\end{tabular}

NPD, no pathologic diagnosis; ADH, atypical duct hyperplasia; DCIS, ductal carcinoma in situ.

(Case 6) in which the information obtained with deeper levels affected clinical management of the patient.

The mammograms and the specimen radiographs of the 50 completely sectioned cases were reviewed. The mammographic calcifications were classified as medium to highly suspicious or as highly suspicious in 20 cases; as low to medium or medium suspicious in 16 cases; and as low suspicious or benign in 12 cases. Mammograms were not available for review in two cases.

On retrospective review of the available $47 \mathrm{spec}-$ imen radiographs, calcifications were identified in the cores in 41 cases. For those cases in which the accession sheet had indicated a positive specimen radiograph, interobserver variation was found in six (accession sheet indicated presence of calcification, but none was found on retrospective review of radiograph).

In those cases with positive specimen radiographs in which calcifications were not found on the first three levels, the rate of identifying calcium after exhaustive sectioning did not vary with the level of radiographic suspicion. Microcalcifications were ultimately found in 6/10 (60\%) of cases labeled medium to high or highly suspicious, $6 / 10$ $(60 \%)$ of cases labeled low to medium or medium suspicious, and 5/8 (63\%) benign or low-suspicious cases.

The cost of exhaustive sectioning compared with preparation of the standard number of sections was analyzed. Technical cost (direct and indirect) for the standard sections in 118 cases (10-month period) was $\$ 7.04$ per case (annual cost $\$ 992.64$ ). Cost for the additional sectioning of 50 cases was $\$ 36.18$ per case, or an increase of $414 \%$ per case and an annual cost of $\$ 2170.80$. These costs are exclusive of the pathologist's direct cost and time for examining the additional sections.

\section{DISCUSSION}

Our comparison of initial and final diagnoses showed that additional histopathologic information 
was gained with deeper levels in 11/50 specimens. In six cases, deeper levels yielded a specific diagnosis or a change that affected clinical management (duct hyperplasia to $\mathrm{ADH}$ ).

There is imperfect correlation between the level of mammographic suspicion and histologic findings. The positive predictive value of mammographic findings based on biopsy yield of cancer has been estimated at $10-40 \%$ (5). In our series of 48 cases with mammographic review and exhaustive histologic sectioning to find the microcalcifications, all of 12 cases that were read as benign or low suspicion at mammography were benign at biopsy. Only 1 of 16 cases (7\%) read as low to medium or medium suspicious, and 3 of 20 (15\%) read as medium to high or highly suspicious, were atypical or malignant on biopsy. For the subset of cases in which the presence of calcification was confirmed on the specimen radiograph and microcalcifications were identified after complete sectioning, the rate of atypia or malignancy was $7 \%$ for cases of low to medium or medium suspicion, and $9 \%$ for cases of medium to high or high suspicion.

Part of the discrepancy between mammography and biopsy can be attributed to sampling error. In addition, calcifications associated with atypia or carcinoma are not always present within the atyp$\mathrm{ical} /$ malignant foci but may be found in adjacent benign ducts or stroma. Correlating biopsy results with the mammographic findings that generated the biopsy is of utmost importance. Dershaw et al. found that $47 \%$ of repeat biopsies in discordant cases yielded a diagnosis of carcinoma (6).

The Joint Task Force of the American College of Radiology, the American College of Surgeons, and the College of American Pathologists recommends that the presence of microcalcifications in SCNB must be confirmed microscopically with deeper levels beyond initial sections being examined if necessary (7). Intuitively, it is the role of the pathologist to identify in the tissue sample the histologic correlate of the clinical finding that prompted the biopsy. In addition, the failure to identify microcalcifications on SCNB performed for that indication usually compels the radiologist to recommend repeat biopsy. The repeat biopsy is usually a surgical one, which markedly raises the cost of the patient's workup. But does exhaustive search for microcalcifications in SCNB yield information beyond that identified on standard levels? The answer is yes, but the yield is low (12\%) and the cost is high $(414 \%$ increase in technical cost alone per case).

There is no universally accepted standard number of initial sections for handling core needle breast biopsies. In an informal survey of nine academic pathology laboratories, seven reported routinely examining three levels, one uses two levels, and one cuts 20 levels, staining every other one
(W.J. Frable, personal communication). Rosen (8) recommends examination of serial sections at two or more levels depending on the sample size. One study investigating the diagnostic yield associated with examination of prostate needle biopsies recommends three levels to minimize the risk of missing a small focus of carcinoma (9).

No recommendation can be derived from our study regarding an optimum number of additional sections to examine short of exhaustive sectioning until calcifications are found because the number of additional sections required to identify calcifications in our series ranged from 1 to 17 . Dahlstrom et $a l$. reported that three cases of ductal carcinoma in situ in their series of 129 SCNB were not detected on the first 24 sections (4).

If no minimum number of sections can be recommended, what steps can be taken to improve cost effectiveness in CNB for microcalcifications? Radiographic confirmation of the presence of calcium in SCNB samples must be performed by the radiologist and documented for the pathologist. At our institution, the radiologist usually separates those cores containing calcium from cores that do not, thereby facilitating the identification of tissue of greatest concern. However, in 11/41 cases (27\%) with confirmed positive specimen radiograph on retrospective review, $\mathrm{CaP}$ or $\mathrm{CaOx}$ was not identified even after sectioning the entire tissue sample. This suggests that the calcifications may have been lost in processing or in facing the block in preparation for sectioning. On review of the specimen radiographs, occasional calcifications appeared to be floating apart from the tissue, and thus may have been lost before embedding.

Radiography of the paraffin block has been recommended as a way of determining whether calcifications remain in unsectioned tissue (7). In our own experience, interpretation of radiographs of paraffin-embedded tissue is much more difficult for SCNB than for NLB, and uncertainty in the interpretation has led us to abandon this step and proceed directly to complete sectioning. In addition, we found interobserver variation in interpretation of specimen radiographs in $15 \%$ of cases. A similar discrepancy may be expected in interpretation of radiographs of paraffin blocks.

Our series of completely sectioned cases with positive specimen radiographs included 12 biopsies of microcalcifications that were benign or of low suspicion by mammography. This was the only group in which no atypia or malignancy was detected. Although the number of cases is small, this finding supports the recommendation that these cases should be managed by 6 -month follow-up mammography rather than biopsy. As pointed out by the Joint Task Force, the cost associated with 
SCNB in these patients may offset the overall cost effectiveness of SCNB compared with NLB (7).

We strongly recommend direct communication between pathologist and radiologist. The specimen accession sheet must include documentation of the presence or absence of calcium on the specimen radiograph. The level of suspicion based on the mammogram should also be indicated. In cases in which the specimen radiograph is negative or equivocal for the presence of calcifications, our results suggest that additional sections beyond three levels yield additional information in a minority of cases. However in individual cases, the additional findings may be important for patient management. In all cases, the pathologic diagnosis must be correlated with the mammographic findings.

\section{REFERENCES}

1. Howisey RL, Acheson MB, Rowbotham RK, Morgan A. A comparison of Medicare reimbursement and results for various imaging-guided breast biopsy techniques. Am J Surg 1997; 173:395-8.
2. Owings DV, Hann L, Schnitt SJ. How thoroughly should needle localization breast biopsies be sampled for microscopic examination? A prospective mammographic/pathologic correlative study. Am J Surg Pathol 1990;14(6):578-83.

3. Dahlstrom JE, Jain S, Sutton T, Sutton S. Diagnostic accuracy of stereotactic core biopsy in a mammographic breast cancer screening programme. Histopathology 1996;28:421-7.

4. Dahlstrom JE, Sutton S, Jain S. Histologic-radiologic correlation of mammographically detected microcalcification in stereotactic core biopsies. Am J Surg Pathol 1998;22(2):256-9.

5. Bassett LW, Liu T-H, Giuliano AE, Gold RH. The prevalence of carcinoma in palpable vs. impalpable, mammographically detected lesions. AJR Am J Roentgenol 1991;157:21-4.

6. Dershaw DD, Morris EA, Liberman L, Abramson AF. Nondiagnostic stereotaxic core breast biopsy: results of rebiopsy. Radiology 1996;198:323-5.

7. Bassett L, Winchester DP, Caplan RB, Dershaw DD, Dowlatshahi K, Evans WP, et al. Stereotactic core-needle biopsy of the breast: a report of the Joint Task Force of the American College of Radiology, American College of Surgeons, and College of American Pathologists. CA Cancer J Clin 1997;47:17190.

8. Rosen PP. Breast pathology: diagnosis by needle core biopsy. Philadelphia: Lippincott Williams \& Wilkins;1999.

9. Lane RB, Lane CG, Mangold KA, Johnson MH, Allsbrook WC. Needle biopsies of the prostate. What constitutes adequate histologic sampling? Arch Pathol Lab Med 1998;122:833-5. 